\title{
Review of: "Scoping review and interpretation of myofascial pain/fibromyalgia syndrome: An attempt to assemble a medical puzzle"
}

\author{
Elyse M Cornett ${ }^{1}$ \\ 1 LSU Health Shreveport
}

Potential competing interests: The author(s) declared that no potential competing interests exist.

The manuscript Scoping review and interpretation of myofascial pain/fibromyalgia syndrome: An attempt to assemble a medical puzzle highlights the importance of why physicians should be aware of this disease and how to treat it appropriately. Throughout the paper, the author focuses on the root cause of myofascial pain by looking at different potential mechanisms as the pathophysiologic process is still not understood completely.

Title: The addition of "A" before "Scoping" should be considered.

Abstract: The author used PRIMSA-ScR guidelines, a 22 item checklist compiled by 24 professionals that specialize in research. The guideline's purpose is to aid researchers in compiling pertinent information for scope reviews. By following the PRIMSA-ScR model, the results of this paper are credible. The abstract is concise and sets up what to expect from the systemic review later on.

Introduction: The author emphasized the importance of why the reader should care about Myofascial Pain Syndrome as it cost billions of dollars annually on the medical system.

Methods: In paragraph 1, it is mentioned that systemic reviews, meta-analyses, and RCT's were searched for key words since 2015. A possible explanation on why 2015 was used as cutoff could add benefit to the paper as other pertinent papers may have been published about MPS at earlier dates. In paragraph 2, medical textbooks were mentioned but were not named. Adding specifics on which textbooks in the methods section will only help solidify the data reported in this review.

Results: The author does an excellent job explaining the importance of fascia, how it works, and why it is an important component of the human anatomy. For example, in paragraph 2 of "Biophysical and mechanical functions," the author gives an example of what can occur if the fascial integrity is interrupted, compromising perfusion of blood vessels and nerve function leading to chronic pain. In "Sedentarism" paragraph, it may be better to discuss the effect immobility has on fascia density and chronic pain. Some statements in this paragraph are scientifically backed that can be used later on the discussion. In sentence 2 of paragraph 4 of MPS, switching the word "in" to "related to" before the phrase "pain clinics" may help the sentence flow better. The sentence seems very wordy and it could possibly use a comma after "TrPs." Discussion Part 1: With 799 records included, the sample size is adequate to be confident in the review of this paper. It would be helpful to have an illustration of the stress relaxation curve of fascial tension to 
allow the reader to follow along easier in the physics etiological consideration. Another consideration is to conclude at the end of the same paragraph with "more hyaluronic acid predisposes to an increase in fascial tension and the risk of developing MPS" so the reader can understand the purpose of the paragraph better. In paragraph 2 of pathophysiological considerations, sedentary lifestyle is discussed leading to increased tension down the contractile cycle. What are some examples of sedentary activities the reader should be aware of? Discussing specific examples will help physicians be aware of risk factors in their patients that may lead to MPS. In paragraph 6 of the same section, the author discusses mechanical force and muscle overuse leading to TrP's that can radiate pain. This statement seems to contradict the sedentary lifestyle hypothesis. Is it so that too much activation of fascial tension and too little activation can both lead to MPS? Please make a note if so. What are some medications that can increase the risk of myofascial pain via inflammatory pathways?

Are there specific mechanical reasons or predispositions to cause the synthesis of alpha SMA fibers not relaxing properly? If so, what can be done to aid in preventing further episodes proactively before treatment is needed? However, the author described concise evidence from studies why needling should be used instead of corticosteroid injections to treat MPS and further evaluation of this technique will only further the understanding of MPS.

Part 2: In "metabolism related findings" paragraph, hyperbaric oxygen therapy was noted to ameliorate symptoms of fibromyalgia, has this been studied yet in MPS? The author discusses fibromyalgia since it seems to be interconnected with MPS but any opportunity to correlate it with MPS, I would mention it in Part 2.

In "complete resolution of fibromyalgia" paragraph, many statistics were discussed and it may be beneficial to add a chart for readers to be able to interpret more easily. The author points out possibilities of misdiagnoses of fibromyalgia based on the specialty of the physician, an important point that can skew results. There are contradictions noted in this paragraph involving laparoscopic surgery relieving fibromyalgia symptoms with Roux en Y surgery vs. appendectomies. Are they any other factors that can influence these results? Citing studies without any further explanation on why specific surgeries increase or decrease the pain threshold in fibromyalgia leaves the reader confused. I know a lot is still unknown about fibromyalgia so if the scientific community does not know why yet, making a note of it would be nice here.

The author raises multiple questions throughout part 2 that have not been answered yet, which is great for a scoping review as it can point other physicians/researchers in the right direction to discover these answers. The author explored many possible theories throughout this review to understand the pathophysiologic

Conclusion: Another consideration for the author to look into is discussing Polycystic Ovarian Syndrome to see if there is a connection with MPS. The author states obesity increases the risk of fascial tension whereas estrogen is protective. It would be interesting to see if obesity or estrogen has a stronger effect on fascial integrity. The author reviews the main points he or she has made previously throughout the 
paper and has guided the reader with a framework on what questions need to be explored more in the scientific community.

Figures: I understand the point of the illustrations, but are there any better images to use to highlight what the author is trying to portray? 